\title{
GPRENT Inherited progressive cardiac conduction disorders
}

\author{
Alban-Elouen Baruteau ${ }^{\mathrm{a}, \mathrm{b}}$, Vincent Probst ${ }^{\mathrm{a}}$, and Hugues Abrie/ ${ }^{\mathrm{c}}$
}

\begin{abstract}
Purpose of review
Progressive cardiac conduction disorder (PCCD) is an inherited cardiac disease that may present as a primary electrical disease or be associated with structural heart disease. In this brief review, we present recent clinical, genetic, and molecular findings relating to PCCD.

\section{Recent findings}

Inherited PCCD in structurally normal hearts has been found to be linked to genetic variants in the ion channel genes SCN5A, SCN1B, SCN1OA, TRPM4, and KCNK17, as well as in genes coding for cardiac connexin proteins. In addition, several SCN5A mutations lead to 'cardiac sodium channelopathy overlap syndrome'. Other genes coding for cardiac transcription factors, such as NKX2.5 and TBX5, are involved in the development of the cardiac conduction system and in the morphogenesis of the heart. Mutations in these two genes have been shown to cause cardiac conduction disorders associated with various congenital heart defects.
\end{abstract}

\section{Summary}

PCCD is a hereditary syndrome, and genetic variants in multiple genes have been described to date. Genetic screening and identification of the causal mutation are crucial for risk stratification and family counselling.

\section{Keywords}

arrhythmias, atrio-ventricular block, electrophysiology, genetics, ion channels/membrane transport

\section{INTRODUCTION}

Major advances have recently been made in our understanding of the development and pathophysiology of progressive cardiac conduction disorders (PCCDs) [1,2]. Descriptions of the various genetic backgrounds behind the rare forms of familial PCCDs have also been reported, highlighting genotype-phenotype correlations and providing crucial insights into the molecular determinants of cardiac conduction [3]. This brief review aims to describe recent major advances in our understanding of the clinical, genetic and molecular characteristics of inherited PCCD.

\section{THE CARDIAC CONDUCTION SYSTEM: STRUCTURAL AND FUNCTIONAL COMPONENTS}

The sino-atrial node, the atrio-ventricular node and the His-Purkinje system constitute the cardiac conduction system, which coordinates the initiation and propagation of the electrical impulse in the heart and the synchronous contraction of both ventricles $[3,4]$. Recent studies have shown that the cardiac conduction system has a unique embryological origin that is distinct from that of the working myocardium [5]. This system involves more structures than originally thought, since it englobes the atrio-ventricular rings, a third retroaortic node and the pulmonary and aortic sleeves $[1,6]$.

Recent findings have increased our understanding of the role of cardiac ion channels underlying the generation and propagation of the electrical impulse through the cardiac conduction system. The upstroke and repolarization of the sino-atrial

\footnotetext{
aL'Institut du Thorax, Institut National de la Santé et de la Recherche Médicale (INSERM) Unité Mixte de Recherche (UMR) 1087, Centre National de la Recherche Scientifique (CNRS) UMR 6291, French Reference Center for Inherited Arrhythmias, Nantes University, Nantes, ${ }^{\mathrm{b}}$ Marie Lannelongue Hospital, Department of Pediatric and Congenital Cardiac Surgery, M3C - French Reference Center for Complex Congenital Heart Diseases, Le Plessis Robinson/Paris Sud University, Le Kremlin Bicêtre, Paris, France and ' ${ }^{\mathrm{D}}$ Department of Clinical Research, Swiss National Centre of Competence in Research (NCCR) TransCure, University of Bern, Bern, Switzerland

Correspondence to Dr Hugues Abriel, MD, PhD, University of Bern, Department of Clinical Research Murtenstrasse, 35, 3010 Bern, Switzerland. Tel: +41 31 6320928; fax: +4131 6320946; e-mail: Hugues. Abriel@dkf.unibe.ch
}

Curr Opin Cardiol 2015, 30:33-39

DOI:10.1097/HCO.0000000000000134 


\section{KEY POINTS}

- Genetic factors play an important role in patients with progressive cardiac conduction disorders.

- Recent studies have demonstrated that parents of paediatric patients with cardiac conduction alterations are also prone to conduction defects, demonstrating the high heritability of this trait.

- Mutations in genes encoding cardiac ion channels, ion channel-interacting proteins, cardiac transcription factors and cytoskeletal elements can cause cardiac conduction alterations.

- These recent findings illustrate the importance of genetic screening and genetic counselling in patients and families with cardiac conduction disorders.

node are the result of depolarizing voltage-gated calcium currents and repolarizing delayed rectifier potassium currents, respectively $[7,8]$. Cardiac automaticity is a result of diastolic depolarization, a spontaneous slow depolarization towards the threshold that generates a new action potential [9]. Voltage-gated sodium channels and gap junctions composed of connexin proteins are responsible for the fast propagation of the action potential through the atria, the His bundle, the Purkinje fibre network and the ventricular myocytes [10].

\section{PROGRESSIVE CARDIAC CONDUCTION DISORDER IS AN INHERITED DISORDER}

Clinical and molecular studies have led to the characterization of PCCD as an inherited disease.

\section{First reports: familial pedigree of progressive cardiac conduction disorder}

In 1901, Morquio was the first to notice a familial segregation of cardiac conduction disorders. A genetic background had long been suggested, with reports of familial clusters of isolated heart block segregation in two or more generations [11]. In 1965, Gazes et al. [12] reported segregation of Adams-Stokes seizures and second-degree heart block in four generations of the same family, suggesting a dominant inheritance. In the past decade, the field of molecular genetics has rapidly expanded and provided crucial data on the genetic basis of PCCD $[13,14]$.

\section{Genetic epidemiological approach to progressive cardiac conduction disorder}

On the basis of the hypothesis that, in an area where the population is geographically stable, the offspring of an ancestor affected by a genetic disease remain in this same area during several generations and a major difference in the disease repartition could appear, a genetic epidemiological approach was recently applied by Gourraud et al. [15] to show familial aggregation for PCCD. Using French social security numbers, the authors determined the city of birth of more than 6600 patients implanted with a pacemaker in the western part of France. Upon mapping the frequency of pacemaker implantations for PCCD, they observed a large heterogeneity in disease frequency, from 0.21 to $2.28 \%$, in specific parishes. This study led to the identification of five large families with PCCD, strongly supporting a genetic background for PCCD.

\section{Congenital and childhood non-immune isolated atrio-ventricular block is a genetic disease}

Congenital atrio-ventricular block is a rare disorder with an estimated prevalence of 1 per 20000 live births. Maternal autoimmune disease accounts for $90-99 \%$ of all cases diagnosed before 6 months of age, where the trans-placental passage of maternal anti-Ro/SSA and/or anti-La/SSB auto-antibodies causes irreversible fibrosis of the fetal conduction system, as well as the inhibition of cardiac L-type calcium channels [16]. In the remaining cases in which no obvious cause of atrio-ventricular block can be identified, the heart block is considered to be idiopathic.

In the first large-scale study, looking at the heritability of paediatric idiopathic heart block in a French nationwide cohort, Baruteau et al. $[17,18]$ observed a high degree of inheritance and a strong genetic contribution in the pathogenesis of congenital and childhood non-immune isolated atrio-ventricular block. Nearly $70 \%$ of the patients with an incomplete heart block progressed to a permanent complete one, suggesting a postnatal degenerative process of the conduction tissue $[17,18]$. The authors hypothesized that this process may be genetically determined. To test this hypothesis, parents of the patients were invited to undergo a screening 12-lead ECG. ECG screening in asymptomatic parents of children affected by idiopathic atrio-ventricular block revealed a high prevalence of cardiac conduction alterations characterized by a long P wave and prolonged PR and QRS intervals, indicative of intra-atrial, atrio-ventricular and intraventricular conduction abnormalities. Moreover, well-characterized conduction disturbances (mainly first-degree atrio-ventricular block, right bundle branch block and left axis deviation) were more frequent in parents of the patients of the cohort than in matched healthy control individuals. The 
estimated heritability for isolated conduction disturbances was $91 \%$ and two SCN5A mutations were identified in the affected children, confirming that congenital and childhood non-immune isolated atrio-ventricular block is a highly heritable trait $[18,19]$.

\section{CLINICAL/MOLECULAR DESCRIPTION OF INHERITED PROGRESSIVE CARDIAC CONDUCTION DISORDER IN STRUCTURALLY NORMAL HEARTS}

Progressive cardiac conduction disorder may present as a primary electrical disease.

\section{SCN5A}

Cardiac sodium channel dysfunction caused by mutations in the SCN5A gene leads to a broad spectrum of hereditary arrhythmias with variable phenotypic expression [20]. The phenotypic spectrum ranges from lethal arrhythmias to asymptomatic carriers, and includes Brugada syndrome, cardiac conduction disease and atrio-ventricular block, congenital sick sinus syndrome, atrial standstill, familial atrial fibrillation, dilated cardiomyopathy, congenital long-QT syndrome type 3 (LQT3) and sudden infant death syndrome [21]. The gene SCN5A codes for the main cardiac voltage-gated sodium channel Nav1.5 [20], which is expressed in the conduction system and in the regions surrounding the sino-atrial and the atrio-ventricular nodes [7]. Thus far, at least 16 distinct mutations in SCN5A have been found to cause conduction alterations and block in patients and their families [22]. The vast majority of these mutations, when functionally characterized [23], reduced the sodium current, thereby leading to a loss of function consistent with the slowed cardiac conduction observed in patients.

\section{SCN5A overlap syndrome}

SCN5A mutation carriers tend to exhibit overlapping clinical manifestations of the distinct SCN5Arelated syndromes, which is defined as 'SCN5A overlap syndrome' [24]. In many cases, cardiac conduction is altered in these patients. Recently, Kanter et al. [25] reported ventricular arrhythmias and intra-ventricular conduction delays in young children due to loss-of-function sodium and/or calcium channelopathies. Other recently reported SCN5A mutation-related overlap syndromes included LQT3 and dilated cardiomyopathy [26], LQT3 and early-onset lone atrial fibrillation [27], LQT3 and Brugada syndrome [28], and Brugada syndrome, conduction disease and atrial flutter [29"].

\section{SCN1B}

The cardiac sodium channel protein Nav1.5, which constitutes the pore-forming subunit, has been shown to be part of distinct multi-protein complexes in different membrane compartments of the cardiac cells [30]. Among the proteins interacting directly with Nav1.5, at least four beta-subunits were shown to modulate the expression and function of the sodium channel [31]. Watanabe et al. [14] recently found three pathogenic mutations in the gene SCN1B, which codes for the beta1 subunit of the voltage-gated sodium channel, in families with conduction alterations and, in certain cases, Brugada syndrome. All three mutations were shown to decrease the Nav1.5-mediated current in cellular expression system compared with controls.

\section{SCN10A}

In several large, genome-wide association studies, loci within the SCN10A gene, coding for the voltagegated sodium channel Nav1.8, were found to be associated with atrio-ventricular conduction [32] and Brugada syndrome [33"']. Although the expression of Nav1.8 in cardiac cells is still under debate [34,35], a recent study [36] provided evidence that a cardiac enhancer in SCN10A interacts with and regulates the promoter of SCN5A, thus providing an explanation for how SCN10A genetic variants may affect conduction. The details of these complex regulatory mechanisms are still not well understood, and may also be complicated by the observation that many variants of SCN10A were found to be present in patients with Brugada syndrome [37""].

\section{Connexins}

Cardiac gap junctions are composed of proteins called connexins (Cxs), which form low-resistance channels that allow electrical coupling and intercellular electrical communication [10]. Four connexin isoforms are expressed in the human heart with regional distribution: $\mathrm{Cx} 40$, which forms largeconductance gap junction channels; Cx43, which forms medium-conductance gap junction channels; Cx45, which forms small-conductance gap junction channels; and Cx31.9, which forms ultra-smallconductance gap junction channels [38]. Alterations in the expression of cardiac connexins may cause abnormal activation to spread through the myocardium and lead to conduction disorders. In a recent study, Makita et al. [39] first demonstrated 
a causal relationship between nucleotide substitutions in gene coding for connexin-40 protein and progressive familial heart block. From a screening of 156 probands with progressive familial heart block, they identified a mutation in the gene encoding $\mathrm{Cx} 40$ (Q58L) in one family. Heterologous expression of Cx40-Q58L in connexin-deficient cells resulted in a marked reduction in junctional conductance and the diffuse localization of $\mathrm{Cx} 40$ proteins in the vicinity of the plasma membrane, without the formation of gap junctions. This study, which was the first to demonstrate a germ-line mutation in a connexin gene associated with inherited conduction disorders, emphasizes the importance of $\mathrm{Cx} 40$ in the normal propagation of the electrical impulse in the specialized cardiac conduction system.

\section{TRPM4}

Recently, the transient receptor potential (TRP) channel TRPM4 has been implicated in different cardiac disorders [40]. Kruse et al. [41] described the first TRPM4 mutation, p. E7K, in an Afrikaner family with a familial conduction disorder. The affected individuals were heterozygous carriers of the mutation, which was transmitted in a dominant fashion. In cellular expression systems, mutant TRPM4 channels produce a larger current than their wild-type counterparts, possibly due to an insensitivity to deSUMOylation, thus impairing endocytosis and stabilizing the mutant channels at the cell surface [41]. More recently, three more mutations in TRPM4 were reported in French and Lebanese families with PCCD [42]. Functional experiments expressing these three mutant variants of TRPM4 suggested a similar gain-of-function phenomenon related to altered deSUMOylation. In another recent study [43], an additional six TRPM4 mutations in patients with right bundle branch block and atrioventricular block were identified, but electrophysiological or biochemical studies have yet to be carried out in order to elucidate the potential mechanisms involved. Altogether, these recent studies strongly suggest that the cardiac channel TRPM4 plays a key role in the pathogenesis of genetically determined conduction disorders. It may be that gain-of-function mutant TRPM4 channels lead to cell membrane depolarization in the conduction system, thus reducing the number of available sodium channels and resulting in the observed conduction abnormalities.

\section{KCNK17}

In a recent study [44"] reporting on a PCCD patient with idiopathic ventricular fibrillation, whole exome sequencing identified a missense mutation,
G88R, in the gene KCNK17, which codes for the potassium channel TASK-4. This mutation led to a gain of function of the TASK-4-mediated current and may, similarly to the gain-of-function mechanisms proposed for TRPM4 [40], reduce the availability of the sodium current by depolarizing the membrane of conduction system cells. Importantly, this study also demonstrated the expression of the TASK-4 channel in human Purkinje cells [44"].

\section{CLINICAL AND MOLECULAR DESCRIPTION OF INHERITED PROGRESSIVE CARDIAC CONDUCTION DISORDER ASSOCIATED WITH CONGENITAL HEART DISEASES}

Several genes encoding cardiac transcription factors are essential for the development of the conduction system as well as in cardiac septation and morphogenesis. Recent genetic findings suggest that approximately $10 \%$ of sporadic congenital heart diseases may have de-novo mutations that significantly contribute to the disease process $\left[45,46^{\boldsymbol{*}}, 47\right]$. A molecular pathway including TBX5, NKX2.5 and Id2 genes coordinates the specification of ventricular myocytes into the ventricular conduction system lineage [48]. Moreover, several transcription factors modify gene expression of ion channel proteins that contribute to the electrophysiological properties of the conduction system and govern the contraction of the surrounding myocardium [49]. Mutations in genes encoding for core transcription factors critical for cardiac chamber formation, endocardial cushion remodelling and conduction system development, like NKX2.5 and Tbx5, may lead to progressive cardiac conduction disorders associated with congenital heart diseases [50].

\section{NKX2.5}

The first study identifying mutations in the gene coding for the cardiac-specific homeobox transcription factor NKX2.5 was in four families with secundum atrial septal defects (ASDs) and atrioventricular conduction disorders [51]. Since then, numerous mutations in this transcription factor have been reported with various congenital heart defect phenotypes, such as secundum ASD, tetralogy of Fallot, truncus arteriosus, double-outlet right ventricle, L-transposition of great arteries, interrupted aortic arch and hypoplastic left heart syndrome, with or without conduction disorders [52]. More recently, Wenckebach periodicity, ventricular non-compaction and sudden death have been added to the spectrum of clinical manifestations associated with NKX2.5 mutations [53,54], and experimental studies have confirmed that NKX2.5 
regulates the proliferation of atrial working and conduction myocardium in coordination with Notch pathway [55"'].

\section{Tbx5}

In 1997, Basson et al. [56] screened two families with Holt-Oram syndrome and identified mutations in the gene encoding the T-box transcription factor Tbx5. Holt-Oram syndrome is characterized by radial ray upper limb abnormalities and cardiac septation defects with an autosomal dominant transmission pattern. The septal defects consist of secundum ASD, muscular ventricular septal defects or atrioventricular septal defects and may also be associated with aortic coarctation [57]. Affected patients also present various degrees of conduction disorders, such as sinus bradycardia and atrio-ventricular block, even in the absence of overt structural heart disease [58]. Another member of the T-box family transcription factor, TBX3, is found in close proximity to TBX5 on chromosome 12q24. Mutations in TBX3 cause ulnar-mammary syndrome, and an exceptional case of contiguous deletions of both TBX5 and TBX3 has been recently reported in a patient with features of both HoltOram syndrome and ulnar-mammary syndrome, consisting of bilateral symmetric limb malformations, congenital heart defects and rapidly progressive cardiac conduction disease [59"].

\section{DIAGNOSTIC AND THERAPEUTIC MANAGEMENT OF PATIENTS WITH INHERITED PROGRESSIVE CARDIAC CONDUCTION DISORDER}

Inherited progressive cardiac conduction disease is mainly diagnosed in the presence of unexplained progressive conduction abnormalities in individuals younger than 50 years with structurally normal hearts, in the absence of skeletal myopathies, especially if there is a family history of PCCD. The diagnosis of PCCD in an index patient is based on clinical data (history, family history, 12-lead ECG), and a two-dimensional (2D) echocardiography or cardiac MRI when needed to exclude an underlying congenital heart disease and/or cardiomyopathy. Early-onset PCCD in structurally normal heart should prompt consideration of PCCD genetic testing, particularly if there is a family history of PCCD, pacemaker implant or sudden death [60]. Unfortunately, thus far, there is no genotype-based risk stratification that is available. Clinicians should keep a low threshold for investigating symptoms or ECG findings to determine the need for electrophysiological studies or pacemaker implantation. Management of PCCD patients includes restriction of medications with conduction-slowing properties, as well as active treatment of fever in SCN5A mutation carriers. The Heart Rhythm Society/European Heart Rhythm Association/AsiaPacific Heart Rhythm Society expert consensus statement considers that permanent pacemaker implantation is recommended in PCCD patients with either intermittent or permanent third-degree or high-grade atrio-ventricular block, or symptomatic Mobitz I or II second-degree atrio-ventricular block (class I recommendation). Pacemaker implantation can be useful in PCCD patients with bifascicular block with or without first-degree atrioventricular block (class IIa recommendation) [61]. Targeted genetic screening of first-degree relatives of a mutation-positive PCCD patient is also part of the management, to allow prospective follow-up of asymptomatic mutation carriers.

\section{CONCLUSION}

A large number of genes have been linked to cardiac conduction disorders. It is now clear that cardiac conduction defects are not only associated with aging, but that many complex pathophysiological processes may lead to the occurrence of atrio-ventricular and intra-ventricular block. Many genes and gene networks are involved in the electrophysiological activity of the heart, and it is likely that only a small fraction of these genetic defects have been currently identified. In the future, it could be possible to determine most of the causative genetic alterations and in turn determine the actual proportion of conduction disorders due to genetic defects. Once this first step is achieved, genetic analysis would be part of the routine clinical assessment of patients affected by PCCD. Since the appearance of these conduction alterations is a slow process over time, genetic tests could help to better determine the risk of progression of a cardiac conduction defect and hence determine the best timing for pacemaker implantation. In the longer term, it may be possible, through early identification of patients at risk of developing cardiac conduction defects, notably by familial screening, to develop preventive strategies, using drugs able to slow the development of these potentially fatal disorders.

\section{Acknowledgements}

The authors thank Biotelligences LLC (Lausanne, Switzerland) for their suggestions on the text of this manuscript.

\section{Financial support and sponsorship}

None. 


\section{Conflicts of interest}

There are no conflicts of interest.

\section{REFERENCES AND RECOMMENDED READING}

Papers of particular interest, published within the annual period of review, have been highlighted as:

- of special interest

- of outstanding interest

1. Dobrzynski H, Anderson $\mathrm{RH}$, Atkinson $\mathrm{A}$, et al. Structure, function and clinical relevance of the cardiac conduction system, including the atrioventricular ring and outflow tract tissues. Pharmacol Ther 2013; 139:260-288.

2. Gilbert-Barness E. Conduction defects/cardiomyopathies. Adv Pediatr 2014; 61:127-148.

3. Munshi NV. Gene regulatory networks in cardiac conduction system development. Circ Res 2012; 110:1525-1537.

4. Anderson $\mathrm{RH}$, Yanni J, Boyett MR, et al. The anatomy of the cardiac conduction system. Clin Anat 2009; 22:99-113.

5. Gourdie RG, Harris BS, Bond J, et al. Development of the cardiac pacemaking and conduction system. Birth Defects Res C Embryo Today 2003; 69:4657

6. Yanni J, Boyett MR, Anderson RH, et al. The extent of the specialized atrioventricular ring tissues. Heart Rhythm 2009; 6:672-680.

7. Monfredi O, Dobrzynski $\mathrm{H}$, Mondal $\mathrm{T}$, et al. The anatomy and physiology of the sinoatrial node: a contemporary review. Pacing Clin Electrophysiol 2010; 33:1392-1406

8. Nikolaidou $\mathrm{T}$, Aslanidi OV, Zhang $\mathrm{H}$, et al. Structure-function relationship in the sinus and atrioventricular nodes. Pediatr Cardiol 2012; 33:890899.

9. Barbuti A, Baruscotti M, DiFrancesco D. The pacemaker current: from basics to the clinics. J Cardiovasc Electrophysiol 2007; 18:342-347.

10. Kleber AG, Rudy Y. Basic mechanisms of cardiac impulse propagation and associated arrhythmias. Physiol Rev 2004; 84:431-488.

11. Lynch $\mathrm{HT}$, Mohiuddin $\mathrm{S}$, Sketch $\mathrm{MH}$, et al. Hereditary progressive atrioventricular conduction defect. A new syndrome? JAMA 1973; 225:1465-1470.

12. Gazes PC, Culler RM, Taber $E$, et al. Congenital familial cardiac conduction defects. Circulation 1965; 32:32-34.

13. Schott JJ, Alshinawi $C$, Kyndt $F$, et al. Cardiac conduction defects associate with mutations in SCN5A. Nat Genet 1999; 23:20-21.

14. Watanabe H, Koopmann TT, Le SS, et al. Sodium channel beta1 subunit mutations associated with Brugada syndrome and cardiac conduction disease in humans. J Clin Invest 2008; 118:2260-2268.

15. Gourraud JB, Kyndt F, Fouchard S, et al. Identification of a strong genetic background for progressive cardiac conduction defect by epidemiological approach. Heart 2012; 98:1305-1310.

16. Ambrosi A, Sonesson SE, Wahren-Herlenius M. Molecular mechanisms of congenital heart block. Exp Cell Res 2014; 325:2-9.

17. Baruteau $A E$, Fouchard $S$, Behaghel $A$, et al. Characteristics and long-term outcome of nonimmune isolated atrioventricular block diagnosed in utero or early childhood: a multicentre study. Eur Heart J 2012; 33:622-629.

18. Baruteau $A E$, Behaghel $A$, Fouchard $S$, et al. Parental electrocardiographic screening identifies a high degree of inheritance for congenital and childhood non-immune isolated atrio-ventricular block. Circulation 2012; 126:14691477.

19. Cannon BC, Ackerman MJ. Surprise, surprise: idiopathic, isolated complete atrioventricular block may be heritable. Circulation 2012; 126:14341435.

20. Abriel $\mathrm{H}$. Cardiac sodium channel Nav1.5 and interacting proteins: physiology and pathophysiology. J Mol Cell Cardiol 2010; 48:2-11.

21. Remme CA. Cardiac sodium channelopathy associated with SCN5A mutations: electrophysiological, molecular and genetic aspects. J Physiol 2013; 591:4099-4116.

22. Kovach JR, Benson DW. Conduction disorders and Nav1.5. Cardiol Clin $2014 ; 6: 723-731$

23. Zimmer T, Surber R. SCN5A channelopathies: an update on mutations and mechanisms. Prog Biophys Mol Biol 2008; 98:120-136.

24. Remme CA, Wilde AAM, Bezzina $C R$. Cardiac sodium channel overlap syndromes: different faces of SCN5A mutations. Trends Cardiovasc Med 2008; 18:78-87.

25. Kanter RJ, Pfeiffer R, Hu D, et al. Brugada-like syndrome in infancy presenting with rapid ventricular tachycardia and intraventricular conduction delay. Circulation 2012; 125:14-22.

26. Kwon HW, Lee SY, Kwon BS, et al. Long QT syndrome and dilated cardiomyopathy with SCN5A p.R1193Q polymorphism: cardioverter-defibrillator implantation at 27 months. Pacing Clin Electrophysiol 2012; 35:e243e246.

27. Olesen MS, Yuan L, Liang B, et al. High prevalence of long QT syndromeassociated SCN5A variants in patients with early-onset lone atrial fibrillation. Circ Cardiovasc Genet 2012; 5:450-459.
28. Nakaya H. SCN5A mutations associated with overlap phenotype of long QT syndrome type 3 and Brugada syndrome. Circ J 2014; 78:10611062.

29. Hothi SS, Ara F, Timperley JP. Y1449C SCN5A mutation associated with

- overlap disorder comprising conduction disease, Brugada syndrome, and atrial flutter. J Cardiovasc Electrophysiol 2014. [Epub ahead of print]

An interesting study reporting familial segregation of a SCN5A mutation in a single family with a spectrum of cardiac phenotypes including conduction disease, Brugada syndrome and atrial arrhythmias.

30. Shy D, Gillet L, Abriel H. Cardiac sodium channel Nav1.5 distribution in myocytes via interacting proteins: the multiple pool model. Biochim Biophys Acta 2013; 1833:886-894.

31. Brackenbury WJ, Isom LL. Na+ channel $\beta$ subunits: overachievers of the ion channel family. Front Pharmacol 2011; 2:53.

32. Pfeufer A, van NC, Marciante KD, et al. Genome-wide association study of PR interval. Nat Genet 2010; 42:153-159.

33. Bezzina CR, Barc J, Mizusawa Y, et al. Common variants at SCN5A-SCN10A

and HEY2 are associated with Brugada syndrome, a rare disease with high risk of sudden cardiac death. Nat Genet 2013; 45:1044-1049.

A genome-wide association study of 312 individuals with Brugada syndrome and 1115 controls demonstrating that common genetic variants can have a strong impact on the predisposition to rare diseases.

34. Verkerk AO, Remme CA, Schumacher CA, et al. Functional Nav1.8 channels in intracardiac neurons: the link between SCN10A and cardiac electrophysiology. Circ Res 2012; 111:333-343.

35. Yang T, Atack TC, Stroud DM, et al. Blocking SCN1OA channels in heart reduces late sodium current and is antiarrhythmic. Circ Res 2012;111:322332.

36. van den Boogaard $M, S m e m o ~ S$, Burnicka-Turek $O$, et al. A common genetic variant within SCN10A modulates cardiac SCN5A expression. J Clin Invest $2014 ; 124: 1844-1852$.

37. $\mathrm{Hu} D$, Barajas-Martinez $H$, Pfeiffer $R$, et al. Mutations in SCN10A are

- responsible for a large fraction of cases of Brugada syndrome. J Am Coll Cardiol 2014; 64:66-79.

From a cohort of 150 probands and family members and more than 200 healthy controls, this study identifies SCN10A as a major susceptibility gene for BrS, thus greatly enhancing our ability to genotype and risk stratify probands and family members.

38. Temple IP, Inada S, Dobrzynski $\mathrm{H}$, et al. Connexins and the atrioventricular node. Heart Rhythm 2013; 10:297-304.

39. Makita N, Seki A, Sumitomo N, et al. A connexin 40 mutation associated with a malignant variant of progressive familial heart block type I. Circ Arrhythm Electrophysiol 2012; 5:163-172.

40. Abriel H, Syam N, Sottas V, et al. TRPM4 channels in the cardiovascular system: physiology, pathophysiology, and pharmacology. Biochem Pharmacol 2012; 84:873-881.

41. Kruse M, Schulze-Bahr E, Corfield V, et al. Impaired endocytosis of the ion channel TRPM4 is associated with human progressive familial heart block type I. J Clin Invest 2009; 119:2737-2744.

42. Liu H, El ZL, Kruse M, et al. Gain-of-function mutations in TRPM4 cause autosomal dominant isolated cardiac conduction disease. Circ Cardiovasc Genet 2010; 3:374-385.

43. Stallmeyer B, Zumhagen $\mathrm{S}$, Denjoy I, et al. Mutational spectrum in the $\mathrm{Ca}(2+)$ activated cation channel gene TRPM4 in patients with cardiac conductance disturbances. Hum Mutat 2011; 33:109-117.

44. Friedrich $C$, Rinne $S$, Zumhagen $S$, et al. Gain-of-function mutation in TASK-4

- channels and severe cardiac conduction disorder. EMBO Mol Med 2014; 6:937-951.

Whole-exome sequencing identified mutation in the KCNK17 gene encoding the $\mathrm{K} 2 \mathrm{P}$ potassium channel TASK-4 to be associated with cardiac conduction disorder.

45. Bruneau BG. The developmental genetics of congenital heart disease. Nature 2008; 451:943-948.

46. Zaidi $\mathrm{S}$, Choi $\mathrm{M}$, Wakimoto $\mathrm{H}$, et al. De novo mutations in histone-modifying nenes in congenital heart disease. Nature 2013; 498:220-223.

This important study compares the incidence of de-novo mutations in 362 severe congenital heart disease cases and 264 controls by analysing exome sequencing of parent-offspring trios. Authors find a marked excess of de-novo mutations in histone-modifying genes in congenital heart disease.

47. Bruneau BG, Srivastava D. Congenital heart disease: entering a new era of human genetics. Circ Res 2014; 114:598-599.

48. Moskowitz IP, Kim JB, Moore ML, et al. A molecular pathway including Id2, Tbx5, and Nkx2-5 required for cardiac conduction system development. Cell 2007; $129: 1365-1376$.

49. Sizarov A, Devalla HD, Anderson RH, et al. Molecular analysis of patterning of conduction tissues in the developing human heart. Circ Arrhythm Electrophysiol $2011 ; 4: 532-542$.

50. McCulley DJ, Black BL. Transcription factor pathways and congenital heart disease. Curr Top Dev Biol 2012; 100:253-277.

51. Schott JJ, Benson DW, Basson CT, et al. Congenital heart disease caused by mutations in the transcription factor NKX2-5. Science 1998; 281:108111.

52. McElhinney DB, Geiger E, Blinder J, et al. NKX2.5 mutations in patients with congenital heart disease. J Am Coll Cardiol 2003; 42:1650-1655. 
53. Ouyang $P$, Saarel $E$, Bai $Y$, et al. A de novo mutation in NKX2.5 associated with atrial septal defects, ventricular noncompaction, syncope and sudden death. Clin Chim Acta 2011; 412:170-175.

54. Guntheroth W, Chun L, Patton KK, et al. Wenckebach periodicity at rest that normalizes with tachycardia in a family with a NKX2.5 mutation. Am J Cardiol 2012; 110:1646-1650.

55. Nakashima $Y$, Yanez DA, Touma M, et al. Nkx2-5 suppresses the proliferation

- of atrial myocytes and conduction system. Circ Res 2014; 114:1103-1113.

This study suggests that Nkx2-5 regulates the proliferation of atrial working and

conduction myocardium in coordination with Notch pathway.

56. Basson CT, Bachinsky DR, Lin RC, et al. Mutations in human TBX5 [corrected] cause limb and cardiac malformation in Holt-Oram syndrome. Nat Genet 1997; 15:30-35.

57. Baban A, Pitto L, Pulignani S, et al. Holt-Oram syndrome with intermediate atrioventricular canal defect, and aortic coarctation: functional characterization of a de novo TBX5 mutation. Am J Med Genet A 2014; 164A:14191424.
58. McDermott DA, Fong JC, Basson CT. Holt-Oram syndrome. In Pagon RA, Heal AMA, editors. GeneReviews [Internet]. Seattle, WA: University of Washington; 1993. Available from: http://www.ncbi.nlm.nih.gov/books/NBK1111/.

59. Bogarapu S, Bleyl SB, Calhoun A, et al. Phenotype of a patient with - contiguous deletion of TBX5 and TBX3: expanding the disease spectrum. Am J Med Genet A 2014; 164A:1304-1309.

Authors report on a patient with features of both Holt-Oram syndrome and ulnarmammary syndrome consisting of bilateral symmetric limb malformations, congenital cardiac defects, and rapidly progressive cardiac conduction disease.

60. Ackerman MJ, Priori SG, Willems S, et al. HRS/EHRA expert consensus statement on the state of genetic testing for the channelopathies and cardiomyopathies. Europace 2011; 13:1077-1109.

61. Priori SG, Wilde AA, Horie M, et al. HRS/EHRA/APHRS expert consensus statement on the diagnosis and management of patients with inherited primary arrhythmia syndromes: document endorsed by HRS, EHRA, and APHRS in May 2013 and by ACCF AHA, PACES, and AEPC in June 2013. Heart Rhythm 2013; 10:1932-1963. 